\title{
Learning from the Expectations of Others
}

Jim Granato, Eran Guse and M. C. Sunny Wong

February 2006

CWPE 0605

Not to be quoted without permission 


\title{
Learning from the Expectations of Others*
}

\author{
Jim Granato $^{\dagger} \quad$ Eran Guse G $^{\ddagger} \quad$ M.C. Sunny Wong ${ }^{\S}$
}

February 1, 2006

\begin{abstract}
The assumption of perfectly rational representative agents is now commonly questioned. This paper explores the equilibrium properties of boundedly rational heterogeneous agents. We combine an adaptive learning process in a modified cobweb model within a Stackleberg framework. We assume that there is an asymmetric information diffusion process from leading to following firms. In contrast to a simple cobweb model which has a unique REE, our model may produce multiple restricted perceptions equilibria (RPE). However, a unique and learnable RPE, under certain conditions, can exist in our model. In addition, the following firms' forecasts can confound the leading firms' forecasts - when the following firms misinterpret information coming from the leading firms. We refer this situation to the boomerang effect. We also find that the leading firms' mean squared forecast error can be even larger than that of following firms if the proportion of following firms is sufficiently large in the market.

Keywords: Adaptive Learning; Expectational Stability; Information Diffusion, Cobweb Model, Heterogeneous Expectations JEL classification: C62, D84, E37
\end{abstract}

\section{Introduction}

The rational expectations hypothesis (REH) [Muth (1961); Lucas (1972, 1973)] has revolutionized how economists conceptualize and model economic phenomena. Currently rational expectations (RE) represents a key component in the study of macroeconomic problems [Frydman and Phelps (1983); Haltiwanger and Walman (1985)]. Under RE, agents are assumed to act as if they can take conditional (mathematical) expectations of all relevant variables. However, for all its analytical traction, RE is a rather strong assumption. Sargent (1993), for example, points out that agents with $\mathrm{RE}$ are even more sophisticated than the economist who sets up the economic model.

\footnotetext{
*Earlier versions of this paper were presented at the Annual Meeting of the American Political Science Association, San Francisco, California (August 30-September 2, 2001). We would like to thank Mary Bange, Shankha Chakraborty, George Evans, Andreas Flache, Stephen Haynes, Mark Jones, Sandra Sizer Moore, Mike Munger, Jim Robinson, and Kevin Terada for their comments and insights.

${ }^{\dagger}$ Department of Government, University of Texas, Austin, Texas 78712, USA.

‡Faculty of Economics, University of Cambridge, Cambridge, CB3 9DD, United Kingdom.

$\S$ Department of Economics, University of Southern Mississippi, Hattiesburg, MS 39406, USA.
} 
One line of inquiry has been to determine whether a rational expectations equilibrium (REE) can be achieved under the assumption that agents form expectations using less sophisticated mechanisms [Bray (1982); Bray and Savin (1986); Evans (1983); Evans and Honkapohja (2001)]. This line of inquiry allows agents to achieve the REE within the context of a stochastic (updating) process that is typically represented via least squares learning. Agents use a least squares learning rule and do not initially obtain the REE, but over time they update their forecasts (expectations) as new information becomes available.

In more technical terms, least squares learning is used so that agents update parameters of a forecasting rule - perceived law of motion (PLM) - associated with the stochastic process of the variable in question in an attempt to learn an REE. This process requires a condition establishing convergence to the REE - the E-stability condition. The E-stability condition determines the stability of the equilibrium in which the PLM parameters are adjusted slowly to the implied actual law of motion (ALM) parameters. Evans (1989) and Evans and Honkapohja (1992) show that the mapping from the PLM to the ALM is generally consistent with the convergence to REE under least squares learning. This correspondence is called the E-stability principle. ${ }^{1}$ This principle also possesses additional attributes. If the equilibrium is E-stable, then the RE method may be an appropriate technique for solving long run equilibria. Moreover, E-stability conditions can be an important selection criteria (i.e., determining stable solutions) when a model has multiple equilibria.

In this paper, we extend adaptive learning methods to a scenario involving heterogeneous information levels and social interaction. Prior research linking adaptive learning procedures to heterogeneous information levels has not made use of social interaction. This previous research generally assumes that agents forecast independently and solely gather their own information.

For example, Evans and Honkapohja (1996) relax the assumption of homogeneity and allow for $\mathrm{N}$ different groups of agents who may form different (heterogeneous) expectations. Agents are allowed to have different coefficient estimates in the same structural forecasting rule. They use a general cobwebtype model and show that the E-stability condition remains the same as in the case of a homogeneous expectations learning model. In addition, Giannitsarou (2003) allows heterogeneous adaptive learning in an economy with a homogeneous structure. She finds that different types of heterogeneity may result in different stability conditions compared to homogeneous learning.

Others have relaxed the assumption of representative agents in the learning process [Honkapohja and Mitra (Forthcoming)]. They find that such structural heterogeneity alters E-stability conditions in different macroeconomic models. Finally, Guse (2005a) allows heterogeneity in the use of forecasting models used to form expectations in a model with multiple equilibria. He finds that the E-stability conditions of each equilibrium are determined by the proportion of agents using each forecasting model. Furthermore, the two equilibria exchange stability at the smallest proportion of heterogeneity where the mean squared forecast error (MSE) of the two forecasting models are equal.

Currently, there is no study analyzing how agents' interactions would affect model equilibria. While standard adaptive learning models provide important extensions of the RE framework, the assumption of agent forecast independence

\footnotetext{
${ }^{1}$ See Evans and Honkapohja (2001) for further details of E-stability.
} 
can be relaxed. There is empirical justification for this modification. Information diffusion (or interaction) among different groups of agents is perhaps a natural process especially when people do not interpret the public information in an identical manner [Kandel and Zilberfarb (1999)]. Carroll (2003) finds, for example, statistical evidence of information diffusion that professional inflation forecasts Granger-cause household forecasts to become more accurate. ${ }^{2}$

Against this theoretical and empirical background we modify a standard Muthian cobweb model [Muth (1961)] to allow for both information heterogeneity and information diffusion. We assume a Stackleberg framework, where there are two types of agents - first and second moving firms. The first-moving firms (leading firms) make the initial forecasts of an aggregate price level according to exogenous information in a market while second-moving firms (following firms) form their forecasts based on the forecasts made by the leading firms. Although the following firms obtain the leading firms' forecasts, we assume that they are unable to accurately interpret the content of information because there is some miscommunication between firms. Thus, observational errors due to misinterpretation of leading firms' expectations would naturally occur in the information acquisition process. ${ }^{3}$

With the assumption of social/information interactions, we first examine the uniqueness of the equilibrium in a cobweb model. In contrast to results in a simple cobweb model (without social interaction) which has a unique REE, there may exist multiple restricted perceptions equilibria (RPE) in the "interactive" cobweb model. ${ }^{4}$ However, we show that a unique RPE exists under plausible conditions. Next, we demonstrate that the E-stability conditions for the RPE where there is information diffusion are identical to the conditions under no information diffusion (homogeneous expectations). We also find that the conditions for uniqueness and E-stability are the same. It implies that the unique RPE is the only E-stable solution. More importantly, the E-stability conditions rule out other possible equilibria in the model. Although the information diffusion process has no influence on the E-stability conditions, its degree affects the stochastic (equilibrium) process of the aggregate price level.

Perhaps our most important result is the "boomerang effect," which we define as a situation (equilibrium) in which the inaccurate forecasts of the following firms confound the forecasts of the leading firms. We show that the leading firms fail to learn the REE because of the following firms' misinterpretation(s). Not only is the REE unobtainable, but the MSE for the leading firms is larger than it would be under the REE.

In addition to these findings, we also examine the relation between the size of the boomerang effect and the proportion of leading and following firms in the model. We find that, under certain conditions, the leading firms' MSE can be

\footnotetext{
${ }^{2}$ Information diffusion has been documented in many areas of research. For example, financial economists have studied explanations for herding behavior, in which rational investors demonstrate some degree of behavioral convergence [See Devenow and Welch (1996)]. Most recently studies of monetary economics are exploring how information diffusion influences economic forecasting behavior. The standard monetary view from the "credibility" literature holds that policymakers have superior information to citizens [Romer and Romer (2000)] and hence can choose how much information to disseminate for better stabilization outcomes [See Backus and Driffill (1985); Barro and Gordon (1983)].

${ }^{3}$ The terms "observational errors", "(mis)interpretation errors" and "(mis)communication errors" are used interchangeably in the text.

${ }^{4}$ The RPE concept will be discussed further in the following sections.
} 
even larger than that of following firms if the proportion of following firms is sufficiently large.

This paper is organized as follows. Section 2 introduces the cobweb model which includes interactions between leading and following firms. We also show the condition of the unique RPE. In Section 3, we study the E-stability conditions of the model. Section 4 demonstrates the boomerang effect and Section 5 concludes.

\section{A Simplified Interactive Cobweb Model}

The cobweb model has been used extensively in the macroeconomic and learning literature [See Muth (1961); Arifovic (1994); Evans and Honkapohja (2001); Branch and McGough (Forthcoming)]. It is assumed that there are $n$ firms in a competitive market producing a homogeneous product. The firms produce an optimal quantity of their good to maximize their expected profits in accordance with their (rational or nonrational) expectations of the market price in the next period.

The reduced form of the model can be presented as follows: ${ }^{5}$

$$
y_{t}=\beta E_{t-1}^{*} y_{t}+\gamma x_{t-1}+\eta_{t},
$$

where $y_{t}$ is the price level at time $t, E_{t-1}^{*} y_{t}$ is the expectation (not necessarily rational) of $y_{t}$ formed at the end of time $t-1, x_{t-1}$ presents an exogenous observable which is iid $\left(0, \sigma_{x}^{2}\right)$ and $\eta_{t} \sim$ iid $\left(0, \sigma_{\eta}^{2}\right)$.

We modify the cobweb model into a Stackleberg setup where it contains two types of firms. We assume that a proportion of $1-\mu$ of firms as first-moving (or leading) firms which form expectations of the market price based on the information $\left(x_{t-1}\right)$ observed in the market, where $0 \leq \mu<1$.

Based on the adaptive learning literature, we assume that firms act like econometricians and forecast $y_{t}$ by running least squares regressions of $y_{t}$ based on their past information. According to the above setup, we assume that the leading firms (Type-L firms) makes their forecasts based on the form of minimum state variable (MSV) solution. ${ }^{6}$ The preceived law of motion (PLM) for the Type-L firms is given as:

$$
\begin{aligned}
y_{t} & =b x_{t-1}+\varepsilon_{t}, \\
y_{L, t}^{e} & =b x_{t-1},
\end{aligned}
$$

where $y_{L, t}^{e}$ presents the expectations of $y_{t}$ for the Type-L firms at time $t-1$. The remaining $\mu$ firms are assumed to be second-moving (following) firms which observe the Type-L firms' expectations to form their expectations of market price. However, the following (Type-F) firms may interpret (or even misinterpret) the Type-L firms' expectations differently among themselves. Therefore, the PLM

\footnotetext{
${ }^{5}$ Lucas's (1972) model shares the same reduced form as (1) with $0<\beta<1$. Thus, our findings would also apply in his model.

${ }^{6}$ McCallum $(1983,1999)$ discusses the MSV concept at length, interpreting it as a fundamental solution that includes no bubble or sunspot components. McCallum proposes a solution procedure that generates a unique solution in a very wide class of linear RE models.
} 
for the Type-F firms is:

$$
\begin{aligned}
y_{t} & =c\left(y_{L, t}^{e}+v_{t-1}\right)+\varepsilon_{t}, \\
y_{F, t}^{e} & =c\left(b x_{t-1}+v_{t-1}\right),
\end{aligned}
$$

where $y_{F, t}^{e}$ presents the expectations of $y_{t}$ for the Type-F firms at time $t-1$ and $v_{t-1} \sim$ iid $\left(0, \sigma_{v}^{2}\right)$ represents the observational errors which are uncorrelated with $\varepsilon_{t}$.

It is also intuitively reasonable to believe that firms are not able to obtain the exact information from others. Therefore, we impose a distribution of observational errors, $v_{t-1}$, which indicates the degree of misinterpretation of other firms' actions. ${ }^{7}$ Since the Type-F firms obtain the observed information after the Type-L firms form their expectations, the Type-L firms are not able to revise their expectations according to the ex post observational errors generated by the Type-F firms. ${ }^{8}$

Based on the proportions of the Type-L and Type-F firms, average expectations of the market price is:

$$
E_{t-1}^{*} y_{t}=\mu\left[c\left(b x_{t-1}+v_{t-1}\right)\right]+(1-\mu) b x_{t-1} .
$$

The actual law of motion (ALM) is obtained by substituting average expectations of next period's market price into equation (1):

$$
\begin{aligned}
y_{t} & =[\beta b(\mu(c-1)+1)+\gamma] x_{t-1}+\beta \mu c v_{t-1}+\eta_{t} \\
& =\left(\xi_{b}+\xi_{c} b\right) x_{t-1}+\xi_{c} v_{t-1}+\eta_{t},
\end{aligned}
$$

where $\xi_{b} \equiv \beta(1-\mu) b+\gamma$ and $\xi_{c} \equiv \beta \mu c$. Note that the form of each PLM is inconsistent with the ALM. In this case, the projected ALM (associated with each PLM) is the best description of the process of $y_{t}$ within the class of PLM's considered.

The projected ALM's are obtained by computing the following linear projections:

$$
\begin{aligned}
E\left[x_{t-1}\left(\left(\xi_{b}+\xi_{c} b\right) x_{t-1}+\xi_{c} v_{t-1}+\eta_{t}-T_{b} x_{t-1}\right)\right] & =0 \\
E\left[\left(b x_{t-1}+v_{t-1}\right)\left(\left(\xi_{b}+\xi_{c} b\right) x_{t-1}+\xi_{c} v_{t-1}+\eta_{t}-T_{c}\left(b x_{t-1}+v_{t-1}\right)\right)\right] & =0,
\end{aligned}
$$

where:

$$
y_{t}=T_{b} x_{t-1}+\varepsilon_{t},
$$

${ }^{7}$ Kandel and Zilberfarb (1999) argue that people do not interpret existing information in an identical way. Using Israeli inflation forecast data, they show that the hypothesis of identical-information interpretation is rejected. In addition, Bernanke and Woodford (1997) study "inflation forecast" targeting policy rule where policymakers are assumed to conduct monetary policy by targeting private-sector forecasts of inflation. In their model, they also suggest a similar argument regarding the error misinterpretation by private-sector forecasts. The authors argue that some private-sector agents may be "incompetent" at using their information to produce optimal forecasts (p. 659).

${ }^{8}$ If we assume that Type-F firms, which observe the Type-L firms' expectations, also obtain the exogenous information, $x_{t}$, to form their forecasts, both types of firms (Type-L and TypeF) will obtain the rational expectations equilibrium (REE). This is a relatively standard result in the literature. This can be demonstrated and is available upon request from the authors. 
is the projected ALM associated with the PLM of the Type-L firms and:

$$
y_{t}=T_{c}\left(b x_{t-1}+v_{t-1}\right)+\varepsilon_{t}
$$

is the projected ALM associated with the PLM of the Type-F firms.

The above linear projection suggests that the forecasts associated with the two PLM's must satisfy the least-squares orthogonality condition where the regressors are uncorrelated with the forecast errors. If we assume that $E x v=0$, then this projection gives the following T-mapping from the two PLM's to their associated projected ALM's:

$$
\begin{aligned}
T\left(\begin{array}{l}
b \\
c
\end{array}\right) & =\left(\begin{array}{c}
T_{b}(b, c) \\
T_{c}(b, c)
\end{array}\right)=\left(\begin{array}{c}
\xi_{b}+\xi_{c} b \\
\xi_{c}+\frac{b \sigma_{x}^{2}}{b^{2} \sigma_{x}^{2}+\sigma_{v}^{2}} \xi_{b}
\end{array}\right) \\
& =\left(\begin{array}{c}
{[(1-\mu)+\mu c] \beta b+\gamma} \\
\mu c \beta+\frac{b \sigma_{x}^{2}}{b^{2} \sigma_{x}^{2}+\sigma_{v}^{2}}((1-\mu) \beta b+\gamma)
\end{array}\right) .
\end{aligned}
$$

In a misspecified model, Evans and Honkapohja (2001) refer to the resulting equilibria as "restricted perceptions" equilibria (RPE) ${ }^{9}$ since agents are not fully aware of the true stochastic process of the aggregate variables they are forecasting. The RPE can be found where:

$$
T\left(\begin{array}{l}
b \\
c
\end{array}\right)=\left(\begin{array}{l}
b \\
c
\end{array}\right) .
$$

The RPE is the following in this model:

$$
\begin{aligned}
& \bar{b}=\frac{\gamma}{1-\beta(1-\mu+\mu \bar{c})} \\
& \bar{c}=\frac{\bar{b}^{2} \sigma_{x}^{2}}{\bar{b}^{2} \sigma_{x}^{2}+(1-\beta \mu) \sigma_{v}^{2}} .
\end{aligned}
$$

Although firms misspecify their forecasting models, the RPE are optimal relative to the restricted information set used by the firms. Due to the orthogonality condition, firms cannot detect a misspecification unless they step outside of their forecasting models. This is one limitation of the RPE as variables in the ALM not included in a forecast will be correlated with the forecast errors. As the variable $v_{t-1}$ is unobservable for the Type-L firms, this does not present a problem.

From solution (6), we observe that the RPE of $\bar{b}$ and $\bar{c}$ are non-linear and there may exist multiple equilibria for a open set of parameters when $\sigma_{v}^{2}>0$. However, we show that there is a unique RPE when $\beta<1$ and $\sigma_{v}^{2}>0$. On the other hand, if $\sigma_{v}^{2}=0$, both types of firms would have the same information and therefore the RPE is equivalent to the unique REE where $(\bar{b}, \bar{c})=\left(\frac{\gamma}{1-\beta}, 1\right)$. We summarize the result in the following proposition:

Proposition 1. There exists a unique RPE of $\bar{b}$ and $\bar{c}$ when $\beta<1$.

\footnotetext{
${ }^{9}$ Restricted perceptions are also discussed in Adam (2005), Adam, Evans, and Honkapohja (Forthcoming), Branch and Evans (Forthcoming), and Guse (2005b).
} 
The proof is given in Appendix A. Next, we explore some important properties of the RPE which will prove useful when discussing the learnability of the RPE. The properties are expressed in the following lemma:

Lemma 1. The RPE $\bar{b}$ and $\bar{c}$ have the following properties:

1. $\bar{c} \in(0,1]$ if $\beta<1$ and $\sigma_{v}^{2}$ is finite.

2. $\operatorname{sign}(\bar{b})=\operatorname{sign}(\gamma)$ if $\beta<1$.

3. $\bar{c}$ and $|\bar{b}|$ are monotonically increasing in $\beta$ for $\beta<1$.

The proof is given in Appendix B. Part 1 of Lemma 1 states that the Type-F firms will always use some information from the Type- $\mathrm{L}$ firms in equilibrium. As mentioned above, if $\sigma_{v}^{2}=0$, then the Type-F firms have the same information as the Type-L firms and thus the equilibrium level of $c$ will be $\bar{c}=1$. As $\sigma_{v}^{2}$ increases, the information from the Type-L firms becomes less useful to the Type-F firms and thus $\bar{c} \rightarrow 0$ as $\sigma_{v}^{2} \rightarrow \infty$. Part 2 of the Lemma states that the sign of $\bar{b}$ must be consistent with the sign of $\gamma$ for $\beta<1$. It turns out that if $\beta>1$ this is not always the case. ${ }^{10}$ Finally, part 3 of the Lemma states that the information from the Type-F firms is more useful for larger values of $\beta$ (for a fixed $\sigma_{v}^{2}$ ) when $\beta<1$. An increase in $\beta$ suggests that expectations of $y_{t}$ are more important and this feeds back into the the equilibrium by increasing $|\bar{b}|$. As the useful information $\left(\left|b x_{t-1}\right|\right)$ increases relative to the miscommunication error $\left(v_{t-1}\right)$, the Type-F firms would wish to make more use of the information by increasing $\bar{c}$. Therefore, when $\beta \rightarrow 1$, it turns out that $|\bar{b}| \rightarrow \infty$ and $\bar{c} \rightarrow 1$.

\section{Expectational Stability of the RPE}

Evans and Honkapohja (2001) discuss the E-stability condition of the cobweb model under homogeneous expectations. Assuming that all agents have the forecasting rules as equation (2), they show that the E-stability condition is $\beta<1$. In Evans and Honkapohja (1996), they relax the assumption of homogeneous expectations learning allowing for $\mathrm{N}$ different groups of agents forming different expectations. Based the above set-up, they find the E-stability is the same as that for the case of homogeneous expectations learning. In this section, we further explore the E-stability condition in the cobweb model where we allow for interactions among agents. As mentioned before, there may exist multiple RPE according to solution (6). Interestingly, we find a striking result that the E-stability condition under the process of information diffusion is $\beta<1$ which is also equivalent to that for the cases of homogeneous and heterogeneous expectations learning. It also provides an important implication that only the unique RPE is E-stable. Although there may be multiple equilibria when $\beta \geq 1$, it turns out that none of the possible equilibria would be learnable under ordinary least squares (OLS). That is, provided $\beta<1$, both types of firms learn asymptotically the unique restricted perception equilibrium in the model.

\footnotetext{
${ }^{10}$ For $\beta>1$, there may be multiple RPE where no RPE is stable under learning. This inconsistency in signs between $\bar{b}$ and $\gamma$ may be the reason why $\bar{b}$ is unstable under learning.
} 
To show the E-stability condition, consider the following ordinary differential equation (ODE):

$$
\frac{d \phi}{d \tau}=T(\phi)-\phi
$$

where $T$ is the mapping from the PLM, $\phi$, to the implied ALM, $T(\phi)$ and $\tau$ denotes "notional" or "artificial" time. In this case, $T(\phi)$ is represented by equation (5) and

$$
\phi=\left(\begin{array}{c}
b \\
c
\end{array}\right) .
$$

Evans and Honkapohja (2001) define an equilibrium (fixed point of the ODE) to be E-stable if the ODE is stable when evaluated at the equilibrium values.

Similar to the E-stability condition in Evans and Honkapohja (1996 and 2001) where the REE is E-stable if $\beta<1$ and E-unstable if $\beta>1$, based on the T-mapping represented by equation (5), we are able to show that the E-stability conditions do not change under the above form of heterogeneous expectations in the cobweb model. We summarize this finding in the following proposition:

Proposition 2. The RPE $\bar{b}$ and $\bar{c}$ are E-stable if $\beta<1$ and E-unstable for $\beta>1$.

The proof is given in Appendix C. Guse (2005a) has shown that under a similar situation where heterogeneous expectations come from agents using two different forecasting models, the E-stability condition turns out to be a convex combination of the two E-stability conditions under homogeneous expectations.

To study each RPE under homogeneous expectations, consider the following model similar to the ALM above:

$$
y_{t}=\beta E_{t-1}^{*} y_{t}+\gamma x_{t-1}+\delta v_{t-1}+\eta_{t} .
$$

Suppose that all agents had the Type-L PLM:

$$
y_{t}=b x_{t-1}+\eta_{t} .
$$

The ALM is the following:

$$
y_{t}=(\beta b+\gamma) x_{t-1}+\delta v_{t-1}+\eta_{t},
$$

and the projected T-map is:

$$
T(b)=\beta b+\gamma .
$$

Thus, the E-stability condition for the $\operatorname{RPE} \bar{b}$ is $\beta<1$.

Next, assume that all agents had the Type-F PLM where $\bar{b}$ is some constant:

$$
y_{t}=c\left(\bar{b} x_{t-1}+v_{t-1}\right) .
$$

The ALM is the following:

$$
y_{t}=(\beta c \bar{b}+\gamma) x_{t-1}+(\beta c+\delta) v_{t-1}+\eta_{t}
$$


and the projected T-map is:

$$
T(c)=\beta c+\frac{\bar{b} \gamma \sigma_{x}^{2}+\delta \sigma_{v}^{2}}{\bar{b}^{2} \sigma_{x}^{2}+\sigma_{v}^{2}} .
$$

Thus, the E-stability condition for the $\operatorname{RPE} \bar{c}$ is $\beta<1$. As all convex combinations of these two conditions is always $\beta<1$, the E-stability condition for any $\mu \in[0,1]$ must be $\beta<1$.

\section{The Boomerang Effects}

The previous section shows that the E-stability condition in an interactive cobweb model is identical to the one in the standard cobweb model with homogeneous expectations learning. In this section, we discuss the comparative statics of equilibrium (RPE) and its forecast accuracy (i.e., mean squared error $(\mathrm{MSE})$ ) with respect to the observational errors $\left(v_{t-1}\right)$. We argue that, under the process of information diffusion, the inaccurate forecasts of the Type-F firms would eventually confound the Type-L firms' equilibrium and forecast accuracy such that the Type-L firms fail to obtain the REE and have higher MSE. We refer this consequence as the boomerang effect. We then further examine the relationship between the boomerang effect and the fractions of the Type-L and Type-F firms in the model. We find that the Type-L firms' MSE can be larger than that of Type-F firms if $\mu$ is sufficiently large.

\subsection{The Boomerang Effect on the RPE}

In RPE (6) and Lemma 1, we see that the observational error, $v_{t-1}$, plays a very important role in the model. How much the Type-F firms use the observed expectations from the Type-L firms depends on how accurately the Type-F firms interpret the Type-L firms' expectations. The accuracy is represented by the variance of the observational error, $\sigma_{v}^{2}$. Lemma 1 shows that the RPE $\bar{c}$ is between zero and one depending on the size of $\sigma_{v}^{2}$. If the Type-F firms fully understand and make use of the Type-L firms' expectations, (i.e., $\sigma_{v}^{2}=0$ ), then $\bar{c}=1$. It implies that both types of firms' expectations become homogeneous and therefore they are able to achieve the REE.

We also consider the case of $\sigma_{v}^{2}>0$, where the Type-F firms misinterpret the Type-L firms' expectations. Although the Type-L firms use the existing exogenous observable, $x_{t}$, to form their expectations, the ex post observational error created by the Type-F firms eventually confound the Type-L firms to obtain the REE. We refer this result as the boomerang effect on the RPE which is summarized as the following proposition:

Proposition 3. (Boomerang Effect on the RPE). For a finite $\sigma_{v}^{2}$, the Estable $R P E \bar{b} \in\left(\frac{|\gamma|}{1-\beta(1-\mu)}, \frac{|\gamma|}{1-\beta}\right)$ for $\beta \in[0,1)$ and $\bar{b} \in\left(\frac{|\gamma|}{1-\beta(1-\mu)}, \frac{|\gamma|}{1-\beta}\right)$ for $\beta \in(-\infty, 0)$.

The proof of this proposition is straightforward. According to Proposition $2, c \in(0,1]$ for all finite $\sigma_{v}^{2}$; therefore, from equation (6), we see that $|\bar{b}| \in$ $\left(\frac{|\gamma|}{1-\beta(1-\mu)}, \frac{|\gamma|}{1-\beta}\right)$ for $\beta \in(0,1)$ and $|\bar{b}| \in\left(\frac{|\gamma|}{1-\beta(1-\mu)}, \frac{|\gamma|}{1-\beta}\right)$ if $\beta$ is negative. 
When $\beta=0, \bar{b}=\gamma$. It is the case that the expectations are not involved in the model. The shaded region in Figure 1 shows possible values of $\bar{b}$ for a given $\beta$ when $\gamma$ and $\mu$ are fixed. The lower boundary for $\beta<0$ and the upper boundary for $\beta>0$ represent the REE of $b$ when the Type-F firms accurately observe the forecasts from the Type-L firms (i.e., $\sigma_{v}^{2}=0$ and $c=1$ ) while other boundaries represent a case where the Type-F firms do not use any of the forecasts given by the Type-L firms (i.e., $\sigma_{v}^{2} \rightarrow \infty$ and $c \rightarrow 0$ ). RPE $\bar{b}$ would be located in the shaded region and determined by $\sigma_{x}^{2}$ and $\sigma_{v}^{2}$. If the Type-F firms accurately observe the expectations of the Type-L firms $\left(\sigma_{v}^{2}=0\right)$, the Type-L firms obtain the E-stable REE $\left(\bar{b}^{R E E}\right)$ which lies on the boundaries of the REE. However, if the Type-F firms are unable to perfectly observe the Type-L expectations (a finite $\sigma_{v}^{2}$ ), then $\bar{b}$ for the Type-L firms would move to the inside of the shaded region. It turns out that $|\bar{b}|>\left|\bar{b}^{R E E}\right|$ when $\beta<0$, and $|\bar{b}|<\left|\bar{b}^{R E E}\right|$ when $0<\beta<1$. This result represents the Type-L firms' failure to obtain the REE when the Type-F firms misinterpret the forecasts of the Type-L firms under the process of information diffusion.

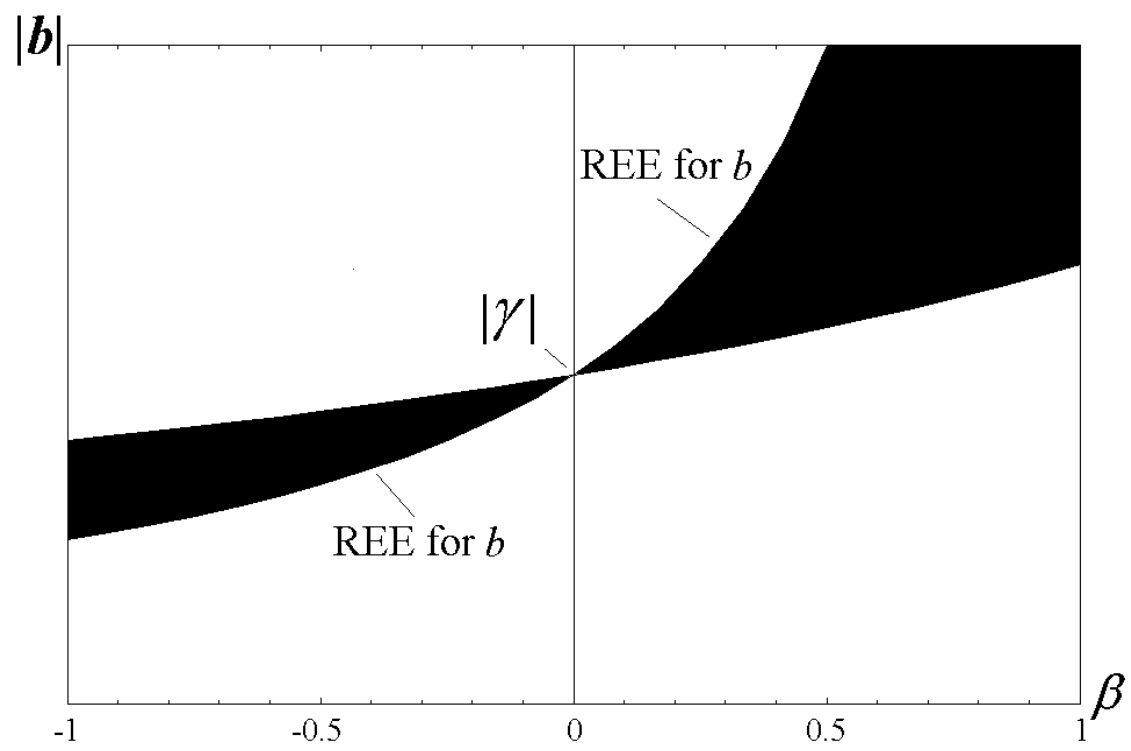

Figure 1. The Region of the Boomerang Effect on the RPE

To illustrate that the Type-L firms fail to obtain the REE with finite values of $\sigma_{v}^{2}$ we simulate the model (1) with the reduced form parameters: $\beta=0.5<1$, $\gamma=2$, and $\mu=0.5$. Our objective of the simulation is to show that firms do not learn the REE but they eventually learn the RPE. The observable $x_{t-1}$ and the unobservable $\eta_{t}$ are assumed to be white noise processes with standard deviation equal to two and one, respectively. We also assume that the Type$\mathrm{L}$ and Type-F firms initially obtain the REE, $\left(b_{0}=4\right.$ and $\left.c_{0}=1\right)$, and the (mis)interpretation error $\left(v_{t-1}\right)$ has a standard deviation of five. We explore whether the parameters will converge to the RPE in the long run. ${ }^{11}$ In this

\footnotetext{
${ }^{11}$ The intuition in the simulation is that Type-F firms perfectly understand the expectations
} 
simulation, the virtual time period is 20,000 .

Figure 2 demonstrates the learning process for the Type-L (upper panel) and the Type-F (lower panel) firms. The Y-axis represents the parameter value of a firm's PLM and the X-axis represents the learning period. To highlight the changes and convergence in the parameters, we separate the time period into three columns. Each column shows a specific time period, ranging from one to 1,000 for column one, 1,000 to 10,000 for column two, and 10,000 to 20,000 for column three.

In Figure 2, we see that, under the process of information diffusion with finite misinterpretation error variance (generated by the Type-F firms), the parameters for both firms PLMs' do not converge to the REE (represented as the dashed lines in the figure). Part 1 of Lemma 1 is shown numerically in the lower panel of Figure 2. With $\sigma_{v}=5$, the Type-F firms make partial use of the expectations formed by the Type-L firm. The result is the Type-F firms' PLM parameter, $c$, converges to RPE $\bar{c}=0.72<1$.

More importantly, the upper panel of Figure 2 describes the boomerang effect on the Type-L firms' forecasts. Although the Type-L firms are initially at the REE and obtain exogenous observables, $x_{t-1}$, to make forecasts, they fail to stay at the REE and instead eventually learn the RPE when they interact with the Type-F firm (with a finite $\sigma_{v}^{2}$ ). For time period between one to 100, the value of $b$ fluctuates and gradually adjusted. After period 4,000, the parameter $b$ becomes more stable and converges to the RPE $\bar{b}=3.56$, which is different from the REE $\bar{b}^{R E E}=4$.

made by Type- $\mathrm{L}$ firms in the early stages of interaction $\left(\sigma_{v}^{2}=0\right)$. Consequently, Type-F firms are able to obtain the REE. However, in subsequent interaction the communication between the two types of firms becomes "noisey" for a variety of reasons and deteriorates so that by time $t=0$ there is a finite (mis)interpretation error $\left(v_{t-1}\right)$ in the information diffusion process. 

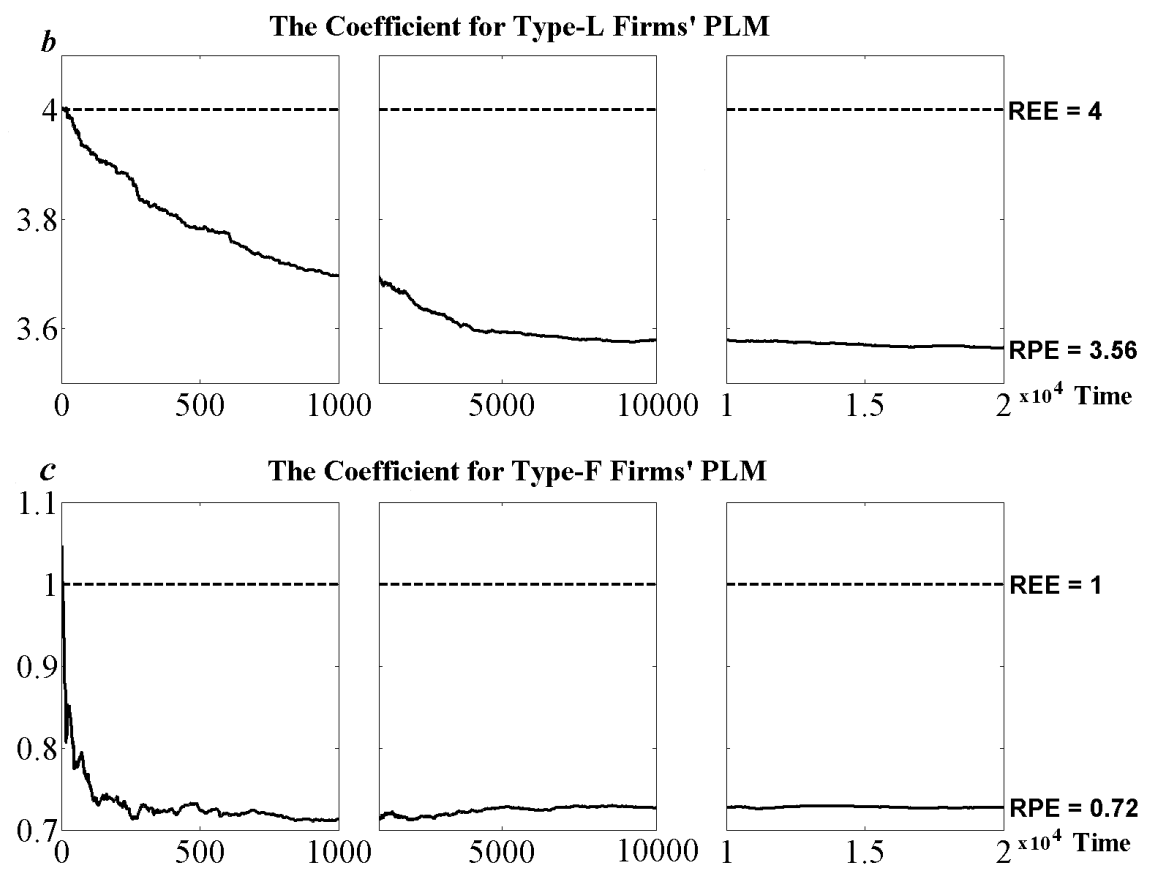

Figure 2. Simulations for Type-L and Type-F Firms' PLM

\subsection{The Boomerang Effect on the MSE}

We also consider how both types of firms' forecast accuracy are affected from miscommunication or misinterpretation of information. To show this, we calculate the MSE for the forecasts of the Type-F and Type-L firms. The MSE for the Type-F firms is given as:

$$
\begin{aligned}
M S E_{F} & =E\left(y_{t+1}-y_{F, t+1}^{e}\right)^{2} \\
& =(1-\bar{c})^{2} \bar{b}^{2} \sigma_{x}^{2}+[(1-\beta \mu) \bar{c}]^{2} \sigma_{v}^{2}+\sigma_{\eta}^{2} .
\end{aligned}
$$

Note that from the equilibrium for $\bar{c}$, we can find an expression for $\bar{b}^{2} \sigma_{x}^{2}$ :

$$
\bar{b}^{2} \sigma_{x}^{2}=\frac{(1-\beta \mu) \bar{c} \sigma_{v}^{2}}{(1-\bar{c})} .
$$

Therefore, we have:

$$
M S E_{F}=\bar{c}(1-\beta \mu)(1-\beta \mu \bar{c}) \sigma_{v}^{2}+\sigma_{\eta}^{2} .
$$


For the Type-L firms, the MSE can be calculated as:

$$
\begin{aligned}
M S E_{L} & =E\left(y_{t+1}-y_{L, t+1}^{e}\right)^{2} \\
& =(\beta \mu \bar{c})^{2} \sigma_{v}^{2}+\sigma_{\eta}^{2}
\end{aligned}
$$

The MSE for the Type-F firms in (7) shows that when the Type-F firms accurately observe the expectations from the Type-L firms $\left(\sigma_{v}^{2}=0\right)$, the Type-F firms obtains the minimum $\operatorname{MSE}\left(M S E_{F}=\sigma_{\eta}^{2}\right)$. However, the finite $\sigma_{v}^{2}$ reduces the Type-F firms' predictive accuracy where $M S E_{F}>\sigma_{\eta}^{2}$. More interestingly, the results for the Type-L firms indicate that only $\sigma_{v}^{2}=0$ or $\sigma_{v}^{2} \rightarrow \infty$ produce the most efficient outcome, $M S E_{L}=\sigma_{\eta}^{2}$. However, if there exists a finite $\sigma_{v}^{2}$, the Type-L firms' forecasts become less efficient (i.e., larger MSE). The consequence is called the boomerang effect on the MSE:

Proposition 4. (The Boomerang Effect on the MSE). The finite variance of the Type-F firms' observational errors $\left(\sigma_{v}^{2}\right)$ generates a higher MSE for the Type-L firms where $M S E_{L}=(\beta \mu \bar{c})^{2} \sigma_{v}^{2}+\sigma_{\eta}^{2}>\sigma_{\eta}^{2}$.

The proof is trivial as it comes directly from $M S E_{L}$. The intuition behind this information rigidity follows from the assumption that Type-L firms are not able to observe the (mis)interpretation error, $v_{t-1}$, when making their forecasts. However, $v_{t-1}$ alters actual $y_{t}$ in equation (4) ; consequently, Type-L firms cannot "disentangle" their expectations from $v_{t-1}$. This results in forecasts by Type-L firms that vary in accuracy (as reflected by larger MSE's).

Next, we also show that the Type-L firms' forecasts can actually have a higher MSE that the Type-F firms' forecasts under certain values of $\mu$. We first present the following proposition:

Proposition 5. $M S E_{L}>M S E_{F}$ if $\beta>0$ and $\bar{c}>\frac{1-\beta \mu}{\beta \mu}$.

The proof is given in Appendix D. This proposition states that it may be possible that the $M S E_{L}>M S E_{F}$ if $\bar{c}$ is large enough. However, this cannot always be the case since $\bar{c}$ is constrained to be between zero and one. The following corollary states that for $M S E_{L}>M S E_{F}$, it must be that $\mu$ and $\beta$ are sufficiently large:

Corollary 1. If $\mu \in(1 / 2,1)$, then there is a $\hat{\beta} \in(1 / 2,1)$ such that if $\hat{\beta}<$ $\beta<1$, then $M S E_{L}>M S E_{F}$.

The proof is given in Appendix E. In Proposition 5 and Corollary 1, we show that when the fraction of the Type-F firms is larger than that of the Type-L firms (i.e., for some $\mu>1 / 2$ ), it is possible that the MSE of the Type-L firms is larger than that of the Type-F firms for some $\beta \in(1 / 2,1)$. We illustrate such scenario in Figures 3 and 4 . By assuming $\mu=0.7>1 / 2$, we obtain a shaded region of $M S E_{L}>M S E_{F}$ under open values of $c$ and $\beta$ which satisfies $c>\frac{1-\beta \mu}{\beta \mu}$, where $\beta$ is on the horizontal axis and $c$ (for any $c$ ) is on the vertical axis. From Proposition 5, we also see that the region becomes larger as $\mu$ 
approaches one.

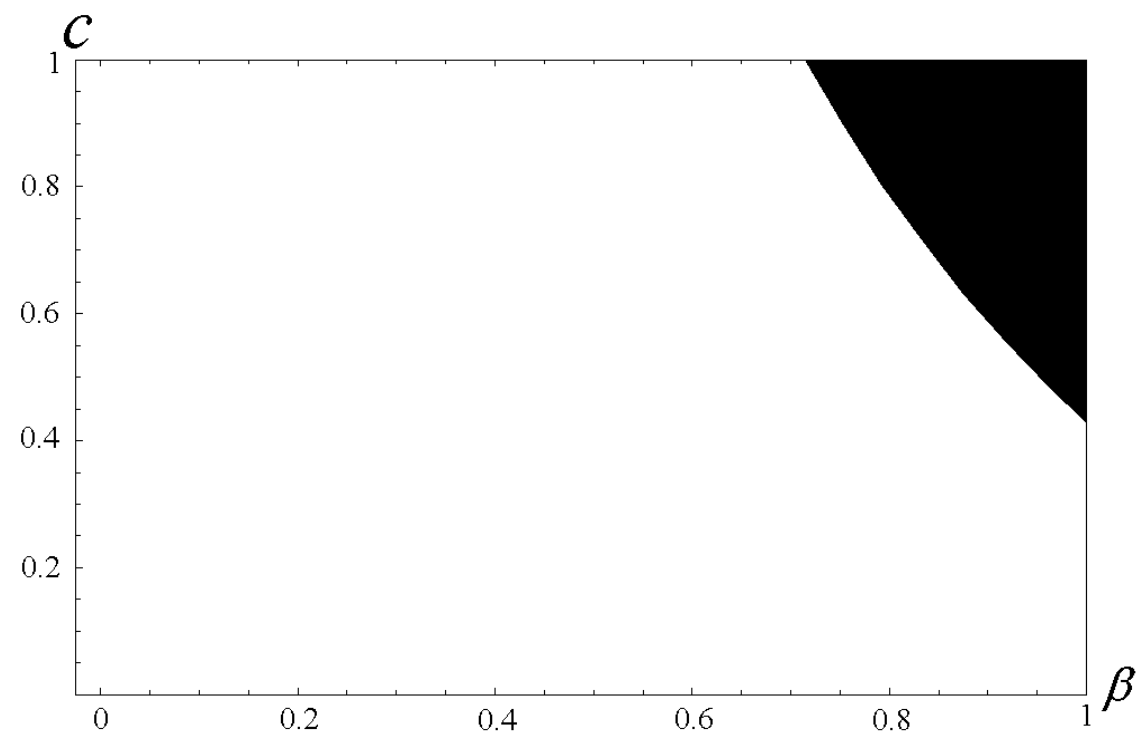

Figure 3. The Region of $M S E_{L}>M S E_{F}$

From Lemma (1), we show that the RPE of $c$ is monotonically increasing in $\beta$. Thus, provided that $\beta$ is large enough, it is possible to have an RPE $\bar{c}$ which lies in the critical region where $M S E_{L}>M S E_{F}$ for some $\mu>1 / 2$. Therefore, in Figure 4, we combine the shaded region with a function of RPE $\bar{c}$ with respect to $\beta .^{12}$ We find that, for a large $\beta$, there exist some values of the $\operatorname{RPE} \bar{c}$ which lie in the shaded region. ${ }^{13}$ In Figure 4, the numerical example shows that the function of RPE intersects the boundary of the shaded region at $\beta=0.74$. It implies that when the proportion of Type-F firms sufficiently outweights that of Type-L firms (i.e., $\mu=0.7$ ), we can obtain a RPE $\bar{c}$ such that $M S E_{L}>M S E_{F}$ for $\beta \in(0.74,1)$. However, when $\mu<0.5$, the critical region does not exist for any $\beta<1$. Therefore, it is impossible to obtain a possible RPE $\bar{c}$ where $M S E_{L}>M S E_{F}$.

\footnotetext{
${ }^{12}$ We assume $\mu=0.7, \sigma_{x}^{2}=1, \sigma_{v}^{2}=2$, and $\gamma=1$.

${ }^{13}$ The discussion of the relationship between $c$ and $\mu$ would be more interesting. However, we are unable to show such relationship analytically as $\bar{b}$ and $\bar{c}$ are non-linearily depended on each other.
} 


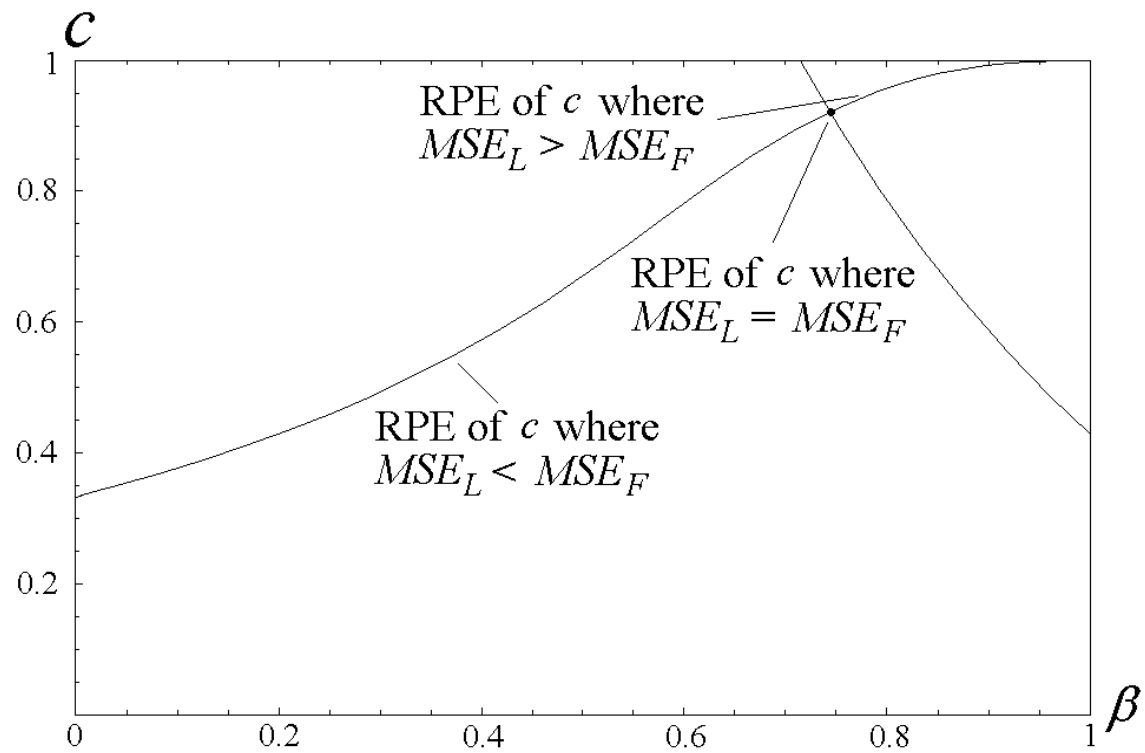

Figure 4. The RPE of $c$ satisfying $M S E_{L}>M S E_{F}$

As noted earlier, the size of the boomerang effect is related to the proportion of the Type-L and Type-F firms. In particular, when the proportion of the Type-F firms becomes large (i.e., a large $\mu$ ), equation (4) shows that the weight of the misinterpretation error $\left(v_{t-1}\right)$ generated by the Type-F firms increases. The variations of $v_{t-1}$ would have a more significant effect (larger inaccuracy) for the Type-L firms' PLM. Eventually, the MSE for the Type-L would turn out be larger than that for the Type-F firms. This would occur when the proportion of the Type-F firms sufficiently exceeds that of the Type-L firms.

\section{Conclusion}

In this paper, we introduce a process of information diffusion in a modified Muthian cobweb model where agents - firms - form their expectations in accordance with an adaptive learning process. We consider two types of firms following a Stackleberg process in the market. The leading firms (Type-L) form initial forecasts while the following firms (Type-F) observe (and use) the leading firms' forecasts when forming their own expectations. In this modified cobweb model, we find there may exist multiple restricted perception equilibria. However, we show that there is a unique RPE under a certain condition (i.e., $\beta<1$ ). In addition, we conclude that, compared to the simple cobweb model, the E-stability condition in the modified model remains unchanged and the unique RPE is the only E-stable solution.

We also focus particular attention on the equilibrium properties and forecasting accuracy of the model. We introduce and find evidence for the boomerang effect, which we define as a situation in which the inaccurate forecasts of the 
Type-F firms confound the Type-L firms' forecasts. We also argue that the MSE's of the Type-L firms can possibly exceed that of the Type-F firms when the proportion of the Type-F firms is larger than the proportion of the Type-L firms.

In the current setting of our model, heterogeneity - the proportion of the Type-L and Type-F firms - is assumed to be exogenous. However, endogenizing heterogeneity would be an important future research challenge [See Brock and Hommes (1997); Evans and Ramey (1998)]. In particular, one issue to consider is the degree to which heterogeneity exists for alternative types of firms if firms optimally choose to become either Type-L or Type-F firms based on the trade-off between forecast accuracy and the cost of acquiring forecast information.

We note that the framework in this paper can be extended to monetary policy issues [See Bernanke and Woodford (1997)]. In particular, there are implications for the overall performance of an inflation-stabilizing monetary policy. If we substitute the public for the Type-F firms and the monetary authority for the Type-L firms and also assume that the information disadvantage resides in the public's limited understanding of economic events, then a plausible consequence (based on our model's findings) is that information diffusion creates a boomerang effect for the policymakers. Since the equilibrium forecasts in an economy are aggregations of agents' forecasts, a large boomerang effect can cause policymakers themselves to make inaccurate forecasts of economic conditions. The inaccurate forecasts can eventually cause additional economic volatility and failed stabilization policies. ${ }^{14}$

To alleviate the boomerang effect, one normative policy suggestion is that policymakers should be more transparent about policy information. Greater transparency will make it possible for the public to better understand how the policy will work and hence make more accurate use of others with more information. $^{15}$ With more precision in information acquisition, the public will confound the policymaker's forecasts less and it can reduce the boomerang effect, improve policy effectiveness, and help with overall economic performance.

\section{Appendices}

\section{Appendix A: Proof of Proposition 1}

If $\sigma_{v}^{2}=0$, it is straightforward to solve a unique equilibrium that $(\bar{b}, \bar{c})=$ $\left(\frac{\gamma}{1-\beta}, 1\right)$. If $\sigma_{v}^{2}>0$, we show the number of real roots in the solution (6) by expanding the cubic function of $\bar{b}$ :

$$
A \bar{b}^{3}+B \bar{b}^{2}+C \bar{b}+D=0
$$

where $A=(1-\beta) \sigma_{x}^{2}, B=-\gamma \sigma_{x}^{2}, C=\sigma_{v}^{2}(1-\beta(1-\mu))(1-\beta \mu), D=$ $-\sigma_{v}^{2} \gamma(1-\beta \mu)$. According to Cardano's approach [See Nickalls (1993)], there exists a single real root in a cubic function if $G^{2}+4 H^{3}>0$, where $G=$ $A^{2} D-A B C / 3+2(B / 3)^{3}$ and $H=A C / 3-(B / 3)^{2}$. We substitute $A, B, C$

\footnotetext{
${ }^{14}$ A similar implication is also suggested by Bomfim (2001).

${ }^{15}$ There is research supporting this common-sense suggestion. Bernanke et al. (1999) notes that when information about the plans, objectives, or decisions of the monetary authorities are carefully explained, the public can more easily understand the contents of a policy announcement.
} 
and $D$ into Cardano's solution, we get:

$$
G^{2}+4 H^{3}=\frac{\left(\sigma_{x}^{2}\right)^{3}}{729}\left(4 M^{3}+N^{2}\right),
$$

where $M=-\gamma^{2} \sigma_{x}^{2}+3 \sigma_{v}^{2}(1-\beta)(1-\beta(1+\beta \mu(1-\mu)))$ and $N=\sigma_{x}^{2} \gamma^{2}\left(2 \sigma_{x}^{2} \gamma^{2}+\right.$ $\left.9 \sigma_{v}^{2}(1-\beta)(1-\beta \mu)(2-\beta(2+\mu))\right)$. Although $M$ can be negative, we can show that, with some manipulations, $4 M^{3}+N^{2}>0$ for $\beta<1$ and $\sigma_{v}^{2}>0$. Since $\bar{b}$ is determined, $\bar{c}$ will be determined in (6).

\section{Appendix B: Proof of Lemma 1}

For 1, consider the RPE for $\bar{c}$ :

$$
\bar{c}=\frac{\bar{b}^{2} \sigma_{x}^{2}}{\bar{b}^{2} \sigma_{x}^{2}+(1-\beta \mu) \sigma_{v}^{2}} .
$$

As $1-\beta \mu>0$ for all $\beta<1$, it must be that $\bar{c} \in(0,1]$. For 2 , consider the RPE for $\bar{b}$ :

$$
\bar{b}=\frac{\gamma}{1-\beta(1-\mu+\mu \bar{c})} .
$$

As $1-\beta(1-\mu+\mu \bar{c})>0$ for $\beta<1, \bar{b}$ and $\gamma$ must have the same sign. Finally, for 3 , one can see that an increase in $\beta$ will increase $|\bar{b}|$ and $\bar{c}$ if $\beta<1$. Therefore, $|\bar{b}|$ and $\bar{c}$ are monotonically increasing in $\beta$.

\section{Appendix C: Proof of Proposition 2}

Consider the standard E-stability ODE:

$$
\frac{\partial \phi}{\partial \tau}=T(\phi)-\phi
$$

where:

$$
\phi=\left(\begin{array}{l}
b \\
c
\end{array}\right) .
$$

Therefore:

$$
\frac{d \phi}{d \tau}=\left(\begin{array}{c}
{[(1-\mu)+\mu c] \beta b+\gamma} \\
\mu c \beta+\frac{b \sigma_{x}^{2}}{b^{2} \sigma_{x}^{2}+\sigma_{v}^{2}}((1-\mu) \beta b+\gamma)
\end{array}\right)-\left(\begin{array}{c}
b \\
c
\end{array}\right) .
$$

Now, consider the ODE for $c$ :

$$
\frac{d c}{d \tau}=\frac{b \sigma_{x}^{2}}{b^{2} \sigma_{x}^{2}+\sigma_{v}^{2}}((1-\mu) \beta b+\gamma)+(\mu \beta-1) c .
$$

Note that $b$ will just affect the fixed point of $\bar{c}$ and will not affect the stability of this ODE. If $b$ is stable, then the fixed point of (11) will be the RPE, and if $b$ is unstable, then the fixed point will be $\bar{c}=1$. Assuming that $b$ is a constant, we can consider (11) as a univariate ODE. The eigenvalue of the Jacobian matrix is the following:

$$
\beta \mu-1
$$


Thus, $\bar{c}$ is E-stable if:

$$
1 \leq \beta<\frac{1}{\mu}
$$

As $c$ is stable for any $b$, we can express the $\operatorname{RPE} \bar{c}$ as a function of $\beta$ as we will wish to determine the value of $c(\beta)$ for a given $\beta$. Next, consider the ODE for $b$ :

$$
\frac{d b}{d \tau}=[(1-\mu)+\mu c(\beta)] \beta b+\gamma-b
$$

If we assume that $c(\beta)$ is a constant for a given $\beta$, then (12) can be considered as a univariate ODE as well. The eigenvalue of the Jacobian is the following:

$$
\beta(1-\mu+\mu c(\beta))-1
$$

Therefore, $\bar{b}$ is E-stable if:

$$
\beta(1-\mu+\mu c(\beta))<1
$$

As $c(\beta)$ is monotonically increasing in $\beta$, there can only be one solution to the equation:

$$
\beta(1-\mu+\mu c(\beta))-1=0 .
$$

Since $c(\beta)$ is a function of $\beta$ and $c(\beta) \rightarrow 1$ as $\beta \rightarrow 1$, the E-stability condition turns out to be:

$$
\beta<1
$$

Therefore, both RPE as E-stable when the more strict condition of $\beta<1$ is satisfied.

\section{Appendix D: Proof of Proposition 5}

Consider the difference between $M S E_{L}$ and $M S E_{F}$ :

$$
\begin{aligned}
M S E_{L}-M S E_{F} & =(\beta \mu \bar{c})^{2} \sigma_{v}^{2}-\bar{c}(1-\beta \mu)(1-\beta \mu \bar{c}) \sigma_{v}^{2} \\
& =\left(-\bar{c}+\beta \mu \bar{c}^{2}+\beta \mu \bar{c}\right) \sigma_{v}^{2}
\end{aligned}
$$

If $\beta>0$, the expression can be positive if:

$$
\bar{c}>\frac{1-\beta \mu}{\beta \mu} .
$$

If $\beta<0$, the expression can be positive if:

$$
\bar{c}<\frac{1-\beta \mu}{\beta \mu}<0 .
$$

Since $\bar{c} \in(0,1]$, this cannot occur.

\section{Appendix E. Proof of Corollary 2}

Let:

$$
c^{*}(\beta)=\frac{1-\beta \mu}{\beta \mu} .
$$

Note that $c^{*}$ is monotonically decreasing in $\beta$ (for $\left.\beta \in(0,1)\right), c^{*}(\beta)<1$ if 
$\beta \mu>1 / 2$, and:

$$
c^{*}(1)=\frac{1-\mu}{\mu} .
$$

Assume that $\mu>1 / 2$, so we have the following:

$$
c^{*}(1)<1 .
$$

According to Lemma (1), we can write the RPE $\bar{c}$ as a monotonically increasing function $\bar{c}(\beta) \in(0,1)$. As $\bar{c}(0)<c^{*}(0)$ and $\bar{c}(1)>c^{*}(1)$, then there will be a $\hat{\beta} \in(1 / 2,1)$ such that:

$$
\bar{c}(\hat{\beta})=c^{*}(\hat{\beta}) .
$$

Therefore, for $\hat{\beta}<\beta<1$, we will have:

$$
\bar{c}>\frac{1-\beta \mu}{\beta \mu},
$$

and thus

$$
M S E_{L}>M S E_{F} .
$$

\section{References}

[1] Adam, K. (2005) 'Learning to Forecast and Cyclical Behavior of Output and Inflation',. Macroeconomic Dynamics 9, 1-27.

[2] Adam, K, G. Evans \& S. Honkapohja (Forthcoming) 'Are Hyperinflation Paths Learnable?', Journal of Economic Dynamics and Control.

[3] Arifovic, J. (1994) 'Genetic Algorithm Learning and the Cobweb Model',. Journal of Economic Dynamics and Control 18, 3-28.

[4] Backus, D. \& J. Driffill (1985) 'Inflation and Reputation',. American Economic Review 75, 530-538.

[5] Barro, R., \& D. Gordon (1983) 'Rules, Discretion and Reputation in a Model of Monetary Policy', Journal of Monetary Economics 1, 101-121.

[6] Bernanke, B., T. Laubach, F. Mishkin \& A. Posen (1999) Inflation Targeting: Lessons from the International Experience. New Jersey: Princeton University Press.

[7] Bernanke, B., and M. Woodford (1997) 'Inflation Forecasts and Monetary Policy', Journal of Money, Credit, and Banking 29, 653-684.

[8] Bomfim, A. N. (2001) 'Heterogeneous Forecasts and Aggregate Dynamics', Journal of Monetary Economics 47, 145-161.

[9] Branch, W. \& B. McGough (Forthcoming) 'Replicator Dynamics in a Cobweb Model with Rationally Heterogeneous Expectations', Journal of Economic Behavior and Organization. 
[10] Branch, W. \& G. Evans (Forthcoming) 'Intrinsic Heterogeneity in Expectation Formation', Journal of Economic Theory.

[11] Brock, W. \& C. Hommes (1997). 'A rational route to randomness', Econometrica 65, 1059-1095.

[12] Bray, M. (1982) 'Learning, Estimation, and the Stability of Rational Expectations', Journal of Economic Theory 26, 318-339.

[13] Bray, M. \& N. E. Savin (1986) 'Rational Expectations, Equilibria, Learning, and Model Specification', Econometrica 54, 1129-1160.

[14] Carroll, C. D. (2002) 'Macroeconomic Expectations of Households and Professional Forecasters', Quarterly Journal of Economics 118, 269-298.

[15] Devenow, A. \& I. Welch (1996) 'Rational Herding in Financial Economics', European Economic Review 40, 603-615.

[16] Evans, G. W. (1983) 'The Stability of Rational Expectations in Macroeconomic Models', In Frydman, R. \& E. Phelps, (ed.), Individual Forecasting and Aggregate Outcomes, New York: Cambridge University Press.

[17] Evans, G. W. (1989) 'The Fragility of Sunspots and Bubbles', Journal of Monetary Economics 23, 297-313.

[18] Evans, G. W. \& S. Honkapohja (1992) 'On the Robustness of Bubbles in Linear RE Models', International Economic Review 33, 1-14.

[19] Evans, G. W. \& S. Honkapohja (1996) 'Least Squares Learning with Heterogeneous Expectations', Economics Letters 53, 197-201.

[20] Evans, G. W. \& S. Honkapohja (2001) Learning and Expectations in Macroeconomics. New Jersey: Princeton University Press.

[21] Evans, G.W., \& G. Ramey (1998) 'Calculation, adaptation and rational expectations', Macroeconomic Dynamics 2, 156-182.

[22] Frydman, R. \& E. Phelps (ed.) (1983) Individual Forecasting and Aggregate Outcomes. Cambridge, UK: Cambridge University Press.

[23] Giannitsarou, C. (2003) 'Heterogeneous Learning', Review of Economic Dynamics 6, 885-906.

[24] Guse, E. (2005a) 'Stability Properties for Learning with Heterogeneous Expectations and Multiple Equilibria', Journal of Economic Dynamics and Control 29, 1623-1642

[25] Guse, E. (2005b) 'Learning in a Misspecified Multivartiate Self-Referential Linear Stochastic Model', Cambridge Working Paper in Economics \#0548.

[26] Haltiwanger, J. \& M. Waldman (1985) 'Rational Expectations and the Limits of Rationality: an Analysis of Heterogeneity', American Economic Review 75, 326-340.

[27] Honkapohja, S. \& K. Mitra (Forthcoming) 'Learning Stability in Economics with Heterogeneous Agents', Review of Economic Dynamics. 
[28] Kandel, E. \& B. Zilberfarb (1999) 'Differential Interpretation of Information in Inflation Forecasts', Review of Economics and Statistics 81, 217-226.

[29] Lucas, R. (1972) 'Expectations and the Neutrality of Money', Journal of Economic Theory 4, 103-124.

[30] Lucas, R. (1973) 'Some International Evidence on Output-Inflation Tradeoffs', American Economic Review 63, 326-334.

[31] McCallum, B. (1983) 'On Nonuniqueness in Linear Rational Expectations Models: An Attempt at Perspective', Journal of Monetary Economics, 11, 134-168.

[32] McCallum, B. (1999) 'Role of the Minimal State Variable Criterion in Rational Expectations Models', International Tax and Public Finance, 6, 621639 .

[33] Muth, J. (1961) 'Rational Expectations and the Theory of Price Movements', Econometrica 29, 315-335.

[34] Nickalls, R. W. D. (1993) 'A New Approach to Solving the Cubic: Cardan's Solution Revealed', The Mathematical Gazette 77, 354-359.

[35] Romer, C. \& D. Romer (2000) 'Federal Reserve Information and the Behavior of Interest Rates', American Economic Review 90, 429-457.

[36] Sargent, T. (1993) Bounded Rationality in Macroeconomics. New York: Oxford University Press. 